\title{
ANGULAR DISTRIBUTIONS FOR KNOCKOUT AND SCATTERING OF PROTONS IN THE EIKONAL APPROXIMATION
}

\author{
A. Bianconi \\ Dipartimento di Chimica e Fisica per i Materiali, Università di Brescia \\ v. Valotti 9, 25133 Brescia, Italy \\ M. Radici \\ Istituto Nazionale di Fisica Nucleare, Sezione di Pavia, \\ v. Bassi 6, 27100 Pavia, Italy
}

\begin{abstract}
The advent of new electron accelerators with few-GeV beam energies makes the $\left(\mathrm{e}, \mathrm{e}^{\prime} \mathrm{p}\right)$ reaction a promising tool for investigating new aspects of the electromagnetic interaction. To this purpose it is crucial to set the scale of Final-State Interactions (FSI) at high ejectile energies. Usually, the problem is faced by mutuating wellestablished results of the Glauber method in the framework of elastic $(p, p)$ scattering. Since the generalization of this eikonal approximation to the $\left(e, e^{\prime} p\right)$ case is not straightforward, we have analyzed the constraints which make the comparison a meaningful one, using the ${ }^{12} \mathrm{C}\left(\mathrm{e}, \mathrm{e}^{\prime} \mathrm{p}\right){ }^{11} \mathrm{~B}_{\mathrm{s} 1 / 2}$ and ${ }^{11} \mathrm{~B}_{\mathrm{s} 1 / 2}(\mathrm{p}, \mathrm{p})$ reactions with outgoing-proton momenta of $4 \mathrm{GeV} / \mathrm{c}$ as a test case. The FSI dominance at large deflection angles produces in the distributions a universal behaviour resembling the coherent diffractive scattering between the ejected proton and the (residual) nucleus. Because of the selected sensitivity of the (e,e'p) distribution to different theoretical ingredients depending on different values of the deflection angle (or transverse missing momentum), it is argued that the previous comparison with elastic proton scattering may represent a convenient tool to disentangle effects due to the (hard) electromagnetic vertex from (exotic) effects related to the propagation of the struck hadron through the nuclear medium.
\end{abstract}




\section{Introduction}

With the advent of new electron accelerators, whose beam energy will range from the few $\mathrm{GeV}$ of CEBAF to the $30 \mathrm{GeV}$ of the planned ELFE setup [四], experiments with electromagnetic probes are expected to reveal new physics, particularly on processes like (e, $\left.\mathrm{e}^{\prime} \mathrm{p}\right)$ scattering [2]. Large missing momenta of the residual nucleus will be available, where the details of long-range correlations (due to the coupling between the motion of the emitten proton and collective surface modes of the residual) and of short-range correlations (due to the strong nucleon-nucleon interaction) are expected to show up in the low and high missing-energy spectrum of the residual, respectively [3]- 8]. In addition, because of the high momentum and energy transferred to the target, new and unexplored features of the electromagnetic hard interaction should appear, which are related, for example, to a proper treatment of relativistic dynamics and off-shellness [9]. Finally, the subsequent propagation of the hadron inside the nuclear medium, usually denoted as Final-State Interactions (FSI), is also a central ingredient of models aiming to describe exotic effects like color transparency [10], if any.

However, while the paucity of data still prevents from putting stringent constraints on the various models for dynamical correlations and/or reaction mechanisms at the interaction vertex [3, 4], the problem of FSI at high projectile energy is usually faced by mutuating well-established results obtained in the framework of elastic proton scattering. In fact, the Glauber approximation [11] has been extensively used in the past years in the analysis of data for (p,p) scattering on complex nuclei [12, 13.

But the generalization to the (e,e'p) scattering is not straightforward, mainly because the kinematics and the state of the initial proton are completely different. Moreover, the validity of this eikonal approximation, based on a completely nonrelativistic formalism, arises from nontrivial cancellations among the leading corrections to the lowest-order theory of elastic scattering [14] and cannot be simply generalized to the inelastic case.

Therefore, after a short review on the general formalism in the framework of the Distorted-Wave Impulse Approximation (DWIA) (Section II), the constraints which make the comparison between FSI in $\left(e, e^{\prime} p\right)$ and $(p, p)$ scattering possible, are addressed in Section III. Firstly, the differences between the two reactions and the choice of the proper form of the optical potential for distorting the outgoing-proton wave function are discussed. Secondly, the 
restrictions on the kinematics and the approximations required to produce similar angular distributions are analyzed. Finally, the selected sensitivity of the results for (e,e'p) scattering to different choices of potentials both for the bound and the scattering states are considered in Section IV. It is shown that at large angles (large values of transverse missing momenta) the FSI are the dominant contribution and produce a typical diffractive tail very sensitive to the nuclear surface.

\section{General formalism}

For the scattering of an ultrarelativistic electron with initial (final) momentum $\boldsymbol{p}_{\mathrm{e}}\left(\boldsymbol{p}_{\mathrm{e}}^{\prime}\right)$, while a nucleon is ejected with final momentum $\boldsymbol{p}^{\prime}$, the six-fold differential cross section in the one-photon exchange approximation reads [15, 16]

$$
\frac{\mathrm{d} \sigma}{\mathrm{d} \boldsymbol{p}_{\mathrm{e}}^{\prime} \mathrm{d} \boldsymbol{p}^{\prime}}=\frac{e^{4}}{8 \pi^{2}} \frac{1}{Q^{4} p_{\mathrm{e}} p_{\mathrm{e}}^{\prime}}\left(\rho_{00} f_{00}+\rho_{11} f_{11}+\rho_{01} f_{01} \cos \alpha+\rho_{1-1} f_{1-1} \cos 2 \alpha\right)
$$

where $Q^{2}=\boldsymbol{q}^{2}-\omega^{2}$ and $\boldsymbol{q}=\boldsymbol{p}_{\mathrm{e}}-\boldsymbol{p}_{\mathrm{e}}^{\prime}, \boldsymbol{\omega}=p_{\mathrm{e}}-p_{\mathrm{e}}^{\prime}$ are the momentum and energy transferred to the target nucleus, respectively. The quantities $\rho_{\lambda \lambda^{\prime}}, f_{\lambda \lambda^{\prime}}$ are expressed on the basis of unit vectors

$$
\begin{aligned}
e_{0} & =(1,0,0,0) \\
e_{ \pm 1} & =\left(0, \mp \sqrt{\frac{1}{2}},-\sqrt{\frac{1}{2}} \mathrm{i}, 0\right),
\end{aligned}
$$

which define the longitudinal $(0)$ and transverse $( \pm 1)$ components of the nuclear response with respect to the polarization of the virtual photon exchanged. The matrix elements $\rho_{\lambda \lambda^{\prime}}$ describe the electrodynamics of the leptonic probe, while $f_{\lambda \lambda^{\prime}}$ depend on $q, \omega, p^{\prime}, \cos \gamma=\boldsymbol{p}^{\prime} \cdot \boldsymbol{q} / p^{\prime} q$, and the dependence on the angle $\alpha$, between the $\left(\boldsymbol{p}^{\prime}, \boldsymbol{q}\right)$ plane and the electron scattering plane, is explicitely put into evidence.

The structure functions $f_{\lambda \lambda^{\prime}}$ are defined in terms of bilinear products of the basic ingredient of the calculation, the scattering amplitude «16

$$
J_{\lambda}(\boldsymbol{q})=\int \mathrm{d} \boldsymbol{r} \mathrm{e}^{\mathrm{i} \boldsymbol{q} \cdot \boldsymbol{r}}\left\langle\Psi_{\mathrm{f}}\left|\hat{J}_{\mu} \cdot \mathrm{e}_{\lambda}^{\mu}\right| \Psi_{\mathrm{i}}\right\rangle
$$


which involves the matrix element of the nuclear charge-current density operator $\hat{J}_{\mu}$ between the initial, $\left|\Psi_{\mathrm{i}}\right\rangle$, and the final, $\left|\Psi_{\mathrm{f}}\right\rangle$, nuclear states. A natural choice for $\left|\Psi_{\mathrm{f}}\right\rangle$ is suggested by the experimental conditions of the reaction selecting a final state which behaves asymptotically as a knocked out nucleon and a residual nucleus in a well defined state with energy $E$ and quantum numbers $a$. By making the same assumption for the initial state, the two specific channels can be projected out of the entire Hilbert space by applying a suitable projection operator [16] to $\left|\Psi_{\mathrm{i}}\right\rangle$ and $\left|\Psi_{\mathrm{f}}\right\rangle$. As a result of space truncation, the scattering amplitude is expressed in a one-body representation in terms of an appropriate effective (one-body) charge-current density operator $\hat{J}_{\mu}^{\mathrm{eff}}$ 16]:

$$
J_{\lambda}(\boldsymbol{q})=\int \mathrm{d} \boldsymbol{r} \mathrm{d} \sigma \mathrm{e}^{\mathrm{i} \boldsymbol{q} \cdot \boldsymbol{r}} \chi_{\mathrm{Ea}}^{(-) *}(\boldsymbol{r}, \sigma) \hat{\mathrm{J}}_{\mu}^{\mathrm{eff}} \cdot \mathrm{e}_{\lambda}^{\mu} \phi_{\mathrm{Ea}}(\boldsymbol{r}, \sigma)\left[\mathrm{S}_{\mathrm{a}}(\mathrm{E})\right]^{1 / 2} .
$$

Here $S_{a}(E)$ is the spectral strength associated with the removal process at the excitation energy $E$ of the residual nucleus; $\phi_{E a}$ is eigenfunction of an energy-dependent Feshbach optical potential referred to the residual at the energy $E ; \chi_{E a}^{(-)}$is eigenfunction of the optical potential at the energy $E+\omega$ and has the boundary conditions of incoming wave. The use of an effective current operator in eq. (4) takes into account effects due to truncation of the Hilbert space and guarantees the orthogonality between $\left|\Psi_{\mathrm{i}}\right\rangle$ and $\left|\Psi_{\mathrm{f}}\right\rangle$ [17.

However, the orthogonality defect is negligible in the standard kinematics for $\left(\mathrm{e}, \mathrm{e}^{\prime} \mathrm{p}\right)$ reactions and $\hat{J}_{\mu}^{\text {eff }}$ is usually replaced by $\hat{J}_{\mu}$ [17], which in turn is approximated by a nonrelativistic expansion in powers of the inverse nucleon mass by means of a Foldy-Wouthuysen canonical transformation [16]. Thus, uncertainties are introduced which depend on the order reached in the nonrelativistic expansion and become more important with increasing energy [9, 18]. But our interest is in the analogies between the phenomenology of FSI in (p,p) and $\left(e, e^{\prime} p\right)$ scattering. Therefore, we have concentrated on the properties of the scattering wave $\chi_{E a}^{(-)}$and we have considered the simplified picture where we retain just the longitudinal component $\hat{J}_{0}$ in the leading order $o(1)$ of the nonrelativistic expansion and we neglect the nucleon form factor. Consequently, the cross section becomes proportional to

$$
\left|\int \mathrm{d} \boldsymbol{r} \mathrm{d} \sigma \mathrm{e}^{\mathrm{i} \boldsymbol{q} \cdot \boldsymbol{r}} \chi_{\mathrm{Ea}}^{(-) *}(\boldsymbol{r}, \sigma) \phi_{\mathrm{Ea}}(\boldsymbol{r}, \sigma)\right|^{2} \equiv \mathrm{S}_{\mathrm{Ea}}^{\mathrm{D}}(\boldsymbol{q})
$$


which is traditionally identified as the "distorted" spectral density $S_{E a}^{\mathrm{D}}$ [19] at the energy $E$ of the residual nucleus with a hole with quantum numbers $a$.

In the framework of the Distorted-Wave Impulse Approximation (DWIA) [15, 16] the socalled spectroscopic amplitudes $\phi_{E a}, \chi_{E a}^{(-)}$are approximated by the solutions of eigenvalue problems with single-particle local energydependent potentials of the Woods-Saxon type. To take into account the nonlocality of the original Feshbach potential, these eigenfunctions are multiplied by the appropriate Perey factor [20]. As for the hole state, in this paper we have considered the potential of Comfort and Karp [21] for ${ }^{12} \mathrm{C}$ with the quantum numbers of the $\mathrm{s} \frac{1}{2}$ shell. The scattering wave function $\chi^{(-)}$is solution of the Schrödinger equation

$$
\left(-\frac{\hbar^{2}}{2 m} \nabla^{2}+V\right) \chi=E_{\mathrm{cm}} \chi,
$$

where $m$ is the reduced mass of the proton in interaction with the residual nucleus, $E_{\mathrm{cm}}$ is its kinetic energy in the $\mathrm{cm}$ system and $V$ contains a local equivalent energy-dependent optical potential effectively describing the residual interaction.

Eq. (6) can be solved for each partial wave of $\chi^{(-)}$up to a maximum angular momentum $L_{\max }\left(p^{\prime}\right)$, which satisfies a convergency criterion. The boundary condition is such that each incoming partial wave coincides asymptotically with the corresponding component of the plane wave associated to the proton momentum $\boldsymbol{p}^{\prime}$. Typically, this method (from now on method A) has been applied to (e,e'p) scattering with proton momenta below $0.5 \mathrm{GeV} / \mathrm{c}$ and $L_{\max }<50$ for a large variety of complex optical potentials, including also spin degrees of freedom [16].

At higher energies the Glauber method [11] suggests an alternative way (from now on method B) of solving eq. (6) by linearizing it along the propagation axis $\hat{z}$ :

$$
\begin{aligned}
\boldsymbol{r} & \equiv z \frac{\boldsymbol{p}^{\prime}}{p^{\prime}}+\boldsymbol{b} \\
\nabla^{2} & \simeq \frac{\partial^{2}}{\partial z^{2}}
\end{aligned}
$$




$$
\begin{aligned}
\left(\frac{\partial^{2}}{\partial z^{2}}+p^{\prime 2}\right) & =\left(\frac{\partial}{\partial z}+\mathrm{ip}^{\prime}\right) \cdot\left(\frac{\partial}{\partial z}-\mathrm{ip}^{\prime}\right) \\
& \simeq 2 \mathrm{ip}^{\prime} \cdot\left(\frac{\partial}{\partial \mathrm{z}}-\mathrm{ip}^{\prime}\right),
\end{aligned}
$$

where $\boldsymbol{b}$ describes the degrees of freedom transverse to the motion of the struck particle with momentum $\boldsymbol{p}^{\prime}$. With this approximation eq. (6) becomes

$$
\left(\frac{\partial}{\partial z}-\mathrm{ip}^{\prime}\right) \chi=\frac{1}{2 \mathrm{ip}^{\prime}} V \chi .
$$

The boundary condition is of incoming unitary flux of plane waves.

\section{Comparison between $\left(e, e^{\prime} p\right)$ and $(p, p)$ scat- tering}

Both methods A and B solve the Schrödinger equation for the nucleon scattering wave. However, they have been traditionally applied to very different reactions, the quasielastic proton knockout and the proton elastic scattering, and different energy ranges. Before addressing the main goal of this paper, i.e. to test the reliability of the Glauber method in $\left(\mathrm{e}, \mathrm{e}^{\prime} \mathrm{p}\right)$ reactions and to deduce information on FSI by comparison with $(\mathrm{p}, \mathrm{p})$ scattering, it is necessary to recall these differences and to point out the conditions required to allow for a meaningful comparison.

\subsection{Differences}

Solving the Schrödinger equation (6) for the scattering state implies that the dynamics is calculated in a nonrelativistic formalism. Relativistic effects are correctly taken into account only in a proper calculation of the kinematics. In the case of the application of the Glauber approach to unpolarized proton-nucleus elastic scattering, this approximation does not seem to produce relevant consequences [12, 13], even if the energies involved would require a priori a fully relativistic treatment. This fact originates from a non trivial cancellation among higher-order corrections to the lowest-order theory [14] and from the observation that the relevant dynamics takes place 
in the transverse plane with respect to the propagation axis $\hat{z}$. On the contrary, a fully relativistic description, for example of both the bound state and the electromagnetic vertex [22, 9, 18, 23], seems to play a significant role in $\left(\mathrm{e}, \mathrm{e}^{\prime} \mathrm{p}\right)$ processes and the different kinematical conditions do not allow for a straightforward generalization of the previous results.

In fact, in ( $p, p)$ reactions the angular distribution of the scattered proton is caused by "soft" diffractive proton-nucleon interactions, assuming that rare hard collisions at very large angles are negligible. In (e,e'p), on the contrary, angular distributions of the emitted-proton momentum $\boldsymbol{p}^{\prime}$ with respect to the direction of the momentum transfer $\boldsymbol{q}$ are possible even in a complete absence of proton-nucleon residual interactions, because of the Fermi motion of the struck proton when considered in its initial bound state.

Moreover, at increasing energies the physical picture implemented by the Glauber method describes a series of "soft" rescatterings between the target nucleons and the projectile, which is approximately considered on-shell. Elastic scattering can be due to diffractive regeneration of the on-shell projectile flux. Also inelastic intermediate states can play a role, but still they are considered on-shell [24]. In the case of proton knockout, the energy and momentum transferred to the target can become very high and the electromagnetic hard vertex can produce a hadron whose nature is quite different from the one of a physical proton. For example, in models of color transparency [25] the possibility is open for the hard production of a hadronic object whose formation length is bigger than the nuclear size: this ejectile is simply unable to further interact during its propagation through the nuclear medium and transforms into an on-shell proton well outside of the nuclear surface. Also from the phenomenology of inclusive electron scattering the suggestion is put forward that intermediate states with small-mass off-shell nucleons are produced by the electromagnetic interaction [26].

To describe this "exotic" behaviour of the ejectile it is necessary to keep under control the details of its whole scattering wave function. Despite of the ambiguities in the optical potential $V$ at small $r$ (related to the limits of models for the nucleon-nucleon interaction at very short distances), the whole spatial range of $\chi^{(-)}(\boldsymbol{r}, \sigma)$ enters the scattering amplitude of eq. (4), or alternatively the distorted spectral density of eq. (5). Instead, specific assumptions in the Glauber approach allow for the calculation of the angular distribution for elastically scattered protons without the need of knowing all the details of the projectile wave function $\Psi(\boldsymbol{r}) \equiv \Psi(r, \theta)$ [1]. Experimental results give 
information on the asymptotic angular distribution of the scattered-proton flux with respect to the incoming one, i.e. give experimental check only for theoretical calculations of the ratio $\left|\Psi(r \rightarrow \infty, \theta) / \Psi\left(r \rightarrow \infty, 180^{\circ}\right)\right|^{2}$.

Nevertheless, the comparison with experimental (p,p) angular distributions has been quite successful for a large selection of target nuclei [13]. While in the case of the only available data for (e,e $\left.e^{\prime} p\right)$ at high energies, taken by the NE18 collaboration [27], the application of the Glauber model in

its most straightforward form leads to an overestimation of the damping of the outgoing-proton flux at small angles. Several interpretations have been proposed to account for this discrepancy [28]-[31]. Here, we would like to focus on the features of the distorting potential $V(r)$. Since the Glauber approach itself is equivalent to the eikonal approximation of method B only for a certain class of potentials, a preliminary requirement for any meaningful comparison is the proper choice of $V(r)$ in eq. (6) and eq. (10).

\subsection{Choice of the distorting potential}

In the Glauber model $V(r)$ is determined in a parameter-free way starting from the elementary free proton-nucleon scattering amplitudes at the considered energy [11]. In DWIA calculations of $\left(e, e^{\prime} p\right)$ in quasielastic conditions, it has usually a Woods-Saxon form whose parameters are fixed by fitting the phase-shifts and the analyzing power of elastic (inelastic) (p,p) scattering on the corresponding residual nucleus [21].

In order to set up a potential which can be equivalently used with methods A and B, the energy range available to the final proton has to be selected. The reliability of the eikonal approximation is supposed to increase with increasing ejectile energy [11], ideally in the limit where $\chi^{(-)}$is expanded on an infinite number of partial waves. On the other hand, method A can be considered reliable only for nucleon energies such that the condition $L_{\max } \gg$ $R_{\text {target }} p^{\prime}$ is fulfilled, with $R_{\text {target }}$ the radius of the target nucleus. Therefore, we have selected outgoing-proton momenta in the intermediate range $1 \leq$ $p^{\prime} \leq 4 \mathrm{GeV} / \mathrm{c}$ and we have solved eq. (6) up to $L_{\max }=120$, which matches the convergency criterion required. $V(r)$ has the simple Woods-Saxon form

$$
V(r)=(U+\mathrm{i} W) \frac{1}{1+\mathrm{e}^{\frac{r-R}{a}}}
$$




$$
\equiv(U+\mathrm{i} W) \rho(r)
$$

with the parameters adjusted for the ${ }^{12} \mathrm{C}$ nucleus, i.e. $R=1.2 \times A^{1 / 3} \mathrm{fm}$ and $a=0.5 \mathrm{fm}$. The nuclear density $\rho(r)$ defined in eq. (111) is normalized such that $\rho(0)=1$. No spin-orbit contribution is taken into account because of the knockout from the s shell of ${ }^{12} \mathrm{C}$.

At the nucleon momenta here considered, the elementary proton-nucleon scattering amplitude is dominated by inelastic processes and $V(r)$ is supposed to be mostly sensitive to the imaginary well depth $W$ [32]. However, no phenomenological phase-shift analysis is available beyond the inelastic threshold, which could constraint $U$ and $W$. In a previous paper [33] we showed that the $S_{\mathrm{s} 1 / 2}^{\mathrm{D}}$ of eq. (5) for the ${ }^{12} \mathrm{C}\left(\mathrm{e}, \mathrm{e}^{\prime} \mathrm{p}\right)$ reaction at $p^{\prime}=q=1.4 \mathrm{GeV} / \mathrm{c}$ and in perpendicular kinematics (i.e. for $\gamma \neq 0$ ) shows a rather clear insensitivity to the sign and magnitude of $U$ for different test choices of $(U, W)$, but for huge values $U \gg W$ which are forbidden by the mainly absorbitive character of the proton-nucleon amplitude at these kinematics. Our conclusion was, therefore, that for $p^{\prime} \geq 1 \mathrm{GeV} / \mathrm{c}$ and confining to perpendicular kinematics one could safely use $U=0$. Our choice is not in contradiction with the Glauber model, where the ratio $U / W$ should equal the ratio between the real and the imaginary parts of the average proton-nucleon forward-scattering amplitude, which is expected to be small above the inelastic threshold [32].

As it is suggested by eq. (10), the Glauber approach predicts $W \propto p^{\prime}$ as far as the proton-nucleon total cross section (and, consequently, the damping of the proton flux) can be considered constant for different choices of $p^{\prime} \simeq q$, i.e. for small angles. We checked [33] that the same property holds, with a good approximation, also for method A, even below the inelastic threshold. However, in order to reproduce the NE18 data, a smaller proportionality factor $W / p^{\prime}$ seems to be required with respect to the one indicated by the Glauber model. Various interpretations have been suggested to explain this discrepancy [28]-[31], whose discussion is beyond the scope of this paper. Here, we adopt the choice $W \propto p^{\prime}$ with a proportionality factor such as to reproduce the NE18 data, i.e. $W=50 p^{\prime} / 1400 \mathrm{MeV}$. This choice is equivalent to retaining the full Glauber method, but assuming a smaller proton-nucleon cross section in nuclear matter than in free space. 


\subsection{Analogies}

For the ${ }^{12} \mathrm{C}\left(\mathrm{e}, \mathrm{e}^{\prime} \mathrm{p}\right)^{11} \mathrm{~B}_{\mathrm{s} 1 / 2}$ reaction at proton momenta in the range $1 \leq p^{\prime} \leq 4$ $\mathrm{GeV} / \mathrm{c}$ we already checked [33, 34 that both methods $\mathrm{A}$ and $\mathrm{B}$ give quite similar angular distribution for $S_{\mathrm{s} 1 / 2}^{\mathrm{D}}$. Particularly at $p^{\prime}=4 \mathrm{GeV} / \mathrm{c}$ [33] the agreement is impressive and suggests that the eikonal approximation of eqs. (7)-(9) is reliable at these energies. A common feature of both methods is that at small angles $\gamma$ (which correspond to missing momenta

$\boldsymbol{p}_{\mathrm{m}} \equiv \boldsymbol{p}^{\prime}-\boldsymbol{q} \lesssim p_{\text {Fermi }}$, with $p_{\text {Fermi }}$ the Fermi momentum of the target nucleus) the distribution is qualitatively dominated by the contribution when no FSI are taken into account, i.e. in the socalled Plane-Wave Impulse Approximation (PWIA). With a good approximation the total result reproduces the single-particle momentum distribution of the struck proton when in its bound state and an additional constant damping. After that threshold, usually around the first diffractive minimum of the distribution, the situation changes completely. By schematically rewriting eq. (5) as

$$
\begin{aligned}
S_{E a}^{\mathrm{D}}(\boldsymbol{q}) & \sim|\mathrm{PWIA}+\mathrm{FSI}|^{2} \\
& =|\mathrm{PWIA}|^{2}+|\mathrm{FSI}|^{2}+2 \operatorname{Re}\left(\mathrm{PWIA} \cdot \mathrm{FSI}^{*}\right)
\end{aligned}
$$

the qualitative picture emerges where for $p_{\mathrm{m}} \sim p_{\text {Fermi }}$ the results start becoming sensitive to the interference between PWIA and FSI and for large angles $\left(p_{\mathrm{m}} \gg p_{\text {Fermi }}\right)$ the $|\mathrm{FSI}|^{2}$ contribution dominates producing an oscillating diffractive pattern which is completely different from the one showed in PWIA (see fig. 3 of ref. [34]). In other words, for very large values of transverse $\boldsymbol{p}_{\mathrm{m}}$ the process can be factorized into the virtual-photon absorption on a free proton and the subsequent coherent diffractive scattering of the struck proton with the residual nucleus. Since the diffractive pattern at large angles is reminiscent of a similar trend in the proton-nucleus elastic scattering [12, it is quite natural to select this kind of kinematics and to try to deduce information on FSI by comparison between the two different reactions.

An expression for (p,p) scattering similar to the distorted spectral density of eq. (5) can be written as

$$
S^{\mathrm{DD}}(\boldsymbol{q}) \equiv\left|\sum_{E a} \int \mathrm{d} \boldsymbol{r} \mathrm{d} \sigma \chi_{\mathrm{f}}^{*}(\boldsymbol{r}, \sigma) \phi_{\mathrm{Ea}}^{*}(\boldsymbol{r}, \sigma) \phi_{\mathrm{Ea}}(\boldsymbol{r}, \sigma) \chi_{\mathrm{i}}(\boldsymbol{r}, \sigma)\right|^{2}
$$




$$
\simeq\left|\int \mathrm{d} \boldsymbol{r} \mathrm{d} \sigma \chi_{\mathrm{f}}^{*}(\boldsymbol{r}, \sigma) \rho(\boldsymbol{r}) \chi_{\mathrm{i}}(\boldsymbol{r}, \sigma)\right|^{2}
$$

where $\chi_{\mathrm{i}}, \chi_{\mathrm{f}}$ are the distorted wave functions for the incoming and outgoing proton flux, respectively, and the sum runs over all the possible discrete states $\phi_{E a}$ with energy $E$ and quantum numbers $a$, that the intermediate proton can form with the target. The $\rho(\boldsymbol{r})$ is, in principle, the diagonal part of the density matrix; in practice, it is approximated by the nuclear density of the target. Eq. (13) is the simplest expression that can be conceived to build the cross section for $(\mathrm{p}, \mathrm{p})$ scattering. Many other corrections have been presented in the literature [13], which would correspond to further improvements in the treatment of FSI in eq. (5), and therefore are disregarded.

Assuming the validity of the eikonal approximation, the density $\rho(r)$ becomes proportional to the potential $V(r)$ entering eq. (10), whose solutions $\chi_{\mathrm{i}}, \chi_{\mathrm{f}}$ are

$$
\begin{aligned}
& \chi_{\mathrm{i}}(\boldsymbol{r})=\mathrm{e}^{\mathrm{i} \boldsymbol{p}_{\mathrm{i}} \cdot \boldsymbol{r}} \mathrm{e}^{\mathrm{C} \int_{-\infty}^{\mathrm{z}} \rho\left(\boldsymbol{r}_{\perp}, \mathrm{z}_{\mathrm{i}}^{\prime}\right) \mathrm{d} \mathrm{z}_{\mathrm{i}}^{\prime}} \\
& \chi_{\mathrm{f}}(\boldsymbol{r})=\mathrm{e}^{\mathrm{i} \boldsymbol{p}_{\mathrm{f}} \cdot \boldsymbol{r}} \mathrm{e}^{\mathrm{C} \int_{\mathrm{z}}^{+\infty} \rho\left(\boldsymbol{r}_{\perp}, \mathrm{z}_{\mathrm{f}}^{\prime}\right) \mathrm{d} \mathrm{z}_{\mathrm{f}}^{\prime}},
\end{aligned}
$$

where $C$ is a constant factor relating $V(r)$ to $\rho(r)$ and $\boldsymbol{r}_{\perp}$ describes the degrees of freedom in the transverse plane with respect to the propagation axis $\hat{z}_{\mathrm{i}}^{\prime}, \hat{z}_{\mathrm{f}}^{\prime}$, which are taken parallel to the momenta $\boldsymbol{p}_{\mathrm{i}}, \boldsymbol{p}_{\mathrm{f}}$ of the incoming and outgoing proton, respectively.

Since at high proton momenta the angular deviation from the initial trajectory is usually small, the integrals in eq. (14) can be computed in the average direction $\hat{z}^{\prime}=\left(\hat{z}_{\mathrm{i}}^{\prime}+\hat{z}_{\mathrm{f}}^{\prime}\right) / 2$. Therefore, eq. (13) becomes

$$
\begin{aligned}
S^{\mathrm{DD}}(\boldsymbol{q}) & =\left|\int \mathrm{d} \boldsymbol{r} \rho(\mathrm{r}) \mathrm{e}^{-\mathrm{i}\left(\boldsymbol{p}_{\mathrm{f}}-\boldsymbol{p}_{\mathrm{i}}\right) \cdot \boldsymbol{r}} \mathrm{e}^{\mathrm{C} \int_{-\infty}^{+\infty} \rho\left(\boldsymbol{r}_{\perp}, \mathrm{z}^{\prime}\right) \mathrm{d} \mathrm{z}^{\prime}}\right|^{2} \\
& \equiv\left|\int \mathrm{d} \boldsymbol{r} \rho(\mathrm{r}) \mathrm{e}^{-\mathrm{i} \boldsymbol{p}_{\mathrm{m}} \cdot \boldsymbol{r}} \mathrm{e}^{\mathrm{C} \int_{-\infty}^{+\infty} \rho\left(\boldsymbol{r}_{\perp}, \mathrm{z}^{\prime}\right) \mathrm{dz} z^{\prime}}\right|^{2}
\end{aligned}
$$

which produces the same results of the Glauber standard expression 11

$$
\int \mathrm{d} \boldsymbol{b} \mathrm{e}^{-\mathrm{i} \boldsymbol{p}_{\mathrm{m}} \cdot \boldsymbol{b}}\left[1-\mathrm{e}^{\mathrm{C} \int_{-\infty}^{+\infty} \rho\left(\boldsymbol{r}_{\perp}, \mathrm{z}^{\prime}\right) \mathrm{dz}}\right] .
$$

Here, $\boldsymbol{p}_{\mathrm{m}}=\boldsymbol{p}_{\mathrm{f}}-\boldsymbol{p}_{\mathrm{i}}$ represents now the difference between the final and initial momenta of the proton, respectively, and is perpendicular to the average 
propagation axis $\hat{z}^{\prime}$. Thus confirming the previous qualitative findings, a meaningful comparison with the $\left(\mathrm{e}, \mathrm{e}^{\prime} \mathrm{p}\right)$ case is possible only for kinematics with large values of transverse missing momenta $p_{\mathrm{m}}=\boldsymbol{p}^{\prime}-\boldsymbol{q}$. In fig. 1 the $S^{\mathrm{DD}}$ of eq. (15) is shown by the long-dashed curve for the ${ }^{11} \mathrm{~B}_{\mathrm{s} 1 / 2}(\mathrm{p}, \mathrm{p})$ reaction at $p_{\mathrm{f}}=4 \mathrm{GeV} / \mathrm{c}$.

By applying the same eikonal approximation to the distorted spectral density for (e,e'p), eq. (5) becomes

$$
\begin{aligned}
S_{\mathrm{s} 1 / 2}^{\mathrm{D}}(\boldsymbol{q}) & =\left|\int \mathrm{d} \boldsymbol{r} \phi_{\mathrm{s} 1 / 2}(\boldsymbol{r}) \mathrm{e}^{-\mathrm{i}\left(\boldsymbol{p}^{\prime}-\boldsymbol{q}\right) \cdot \boldsymbol{r}} \mathrm{e}^{\mathrm{C} \int_{\mathrm{z}}^{+\infty} \rho\left(\boldsymbol{r}_{\perp}, \mathrm{z}^{\prime}\right) \mathrm{dz}}\right|^{2} \\
& \equiv\left|\int \mathrm{d} \boldsymbol{r} \phi_{\mathrm{s} 1 / 2}(\boldsymbol{r}) \mathrm{e}^{-\mathrm{i} \boldsymbol{p}_{\mathrm{m}} \cdot \boldsymbol{r}} \mathrm{e}^{\mathrm{C} \int_{\mathrm{z}}^{+\infty} \rho\left(\boldsymbol{r}_{\perp}, \mathrm{z}^{\prime}\right) \mathrm{dz} z^{\prime}}\right|^{2}
\end{aligned}
$$

The first difference between eq. (17) and eq. (15) is the $z$-dependence of the integral involving the optical potential. If in eq. (17) $p_{\mathrm{m}}$ is chosen to be perpendicular to $\hat{z}$, the Fourier transform will be largely unaffected by the $z$-dependence of the integral and $S_{\mathrm{s} 1 / 2}^{\mathrm{D}}$ can be approximated by

$$
S_{\mathrm{s} 1 / 2}^{\mathrm{D}}(\boldsymbol{q}) \simeq\left|\int \mathrm{d} \boldsymbol{r} \phi_{\mathrm{s} 1 / 2}(\boldsymbol{r}) \mathrm{e}^{-\mathrm{i} \boldsymbol{p}_{\mathrm{m}} \cdot \boldsymbol{r}} \mathrm{e}^{\frac{\mathrm{C}}{2} \int_{-\infty}^{+\infty} \rho\left(\boldsymbol{r}_{\perp}, \mathrm{z}^{\prime}\right) \mathrm{dz} z^{\prime}}\right|^{2} \quad
$$

In fig. 1 the solid and short-dashed curves represent eq. (17) and eq. (18) for the ${ }^{12} \mathrm{C}\left(\mathrm{e}, \mathrm{e}^{\prime} \mathrm{p}\right){ }^{11} \mathrm{~B}_{\mathrm{s} 1 / 2}$ reaction at $p^{\prime}=q=4 \mathrm{GeV} / \mathrm{c}$, respectively. The similarity of the two curves confirms the insensitivity to the longitudinal position of the knockout point $z$. It must be stressed that this is justified only for $\boldsymbol{p}_{\mathrm{m}} \perp \hat{z}$. Assuming that at high energies and momenta the $S_{\mathrm{s} 1 / 2}^{\mathrm{D}}$ is less sensitive to the details of the bound state $\phi_{\mathrm{s} 1 / 2}$ and is dominated by the exponential factors, a very close similarity can be recovered between eq. (15) and eq. (18). The corresponding long-dashed and solid curves in fig. 1 show, after the threshold of the first diffractive minimun where the $|\mathrm{FSI}|^{2}$ contribution in eq. (12) starts dominating, the same universal angular pattern, thus confirming the previous assumption on the FSI dominance at large energies.

The situation can be summarized as follows. In the absence of exotic effects, the FSI for the (e,e'p) reaction become dominant approximately beyond deflection angles $\gamma$ such that the missing momentum $\boldsymbol{p}_{\mathrm{m}}$ exceeds the $p_{\text {Fermi }}$ of the target nucleus. The angular distribution for large values of transverse $\boldsymbol{p}_{\mathrm{m}}$ is completely different from the PWIA result and shows the same universal 
diffractive pattern of the distribution of protons elastically scattered by the same residual nucleus and at the same energy and momentum.

Therefore, since no information was put inside the matrix elements of eq. (17) about the interaction vertex, any deviation from the previous picture has to be ascribed to the details of the (hard) virtual-photon absorption in nuclear medium and to the modifications that can induce on the struck hadron. For example, it has been argued [35] for (e,e'p) that, because of inelastic corrections, at increasing energy a rise of the nuclear transparency is to be expected, that would be hardly distinguishable from effects like color transparency. Since this kind of inelastic corrections is one of the higherorder ingredients adopted to improve the ( $p, p)$ elastic cross section of eq. (13) [24], the comparison between the two reactions in the kinematics above specified could be of much help. In general, the signature of any possible color transparency phenomenon in hard $\left(\mathrm{e}, \mathrm{e}^{\prime} \mathrm{p}\right)$ scattering is that the nuclear response should look more similar to the PWIA result. The traditional strategy has been so far to search for variations of the nuclear damping in the outgoing-proton flux, particularly at small missing momenta [27]. However, very precise and unambiguous results must be obtained to this purpose. From previous comments, it could be equally convenient to analyze the angular distribution for completely exclusive reactions, because FSI can significantly "distort" the PWIA result. Moreover, from the comparison with the diffractive tail of the corresponding elastic $(p, p)$ distribution further insight into the reaction mechanism of the (hard) electromagnetic vertex could be gained.

\section{Properties of FSI for large-angle distribu- tions}

It has already been observed that in the angular distribution for (e,e'p) scattering a special role is played by the $p_{\text {Fermi }}$ of the target nucleus. In fact, for angles corresponding to transverse missing momenta larger than $p_{\text {Fermi }}$ the relation FSI $\gg$ PWIA holds and the shape of the curve is determined by the rescatterings of the hit hadron.

In fig. 2 the $S_{\mathrm{s} 1 / 2}^{\mathrm{D}}$ is shown by the dashed line for the ${ }^{12} \mathrm{C}\left(\mathrm{e}, \mathrm{e}^{\prime} \mathrm{p}\right){ }^{11} \mathrm{~B}_{\mathrm{s} 1 / 2}$ reaction at $p^{\prime}=q=4 \mathrm{GeV} / \mathrm{c}$ with the bound state taken from the solution of the Woods-Saxon potential of Comfort and Karp [21] and with the optical 
potential described in subsection 3.2. The solid curves are produced by varying the well radius $R$ of the optical potential. The small-angle part of the distribution (around the first minimum and following secondary maximum, i.e. for $p_{\mathrm{m}} \lesssim 2 p_{\text {Fermi }}$, which is around $\gamma=6^{\circ}$ for $p^{\prime}=4 \mathrm{GeV} / \mathrm{c}$ ) is not very much affected, while the large-angle diffractive pattern is significantly modified both in the size and in the frequency of the secondary maxima. On the contrary, no significant change is observed when keeping everything fixed but the imaginary depth $W$ in eq. (11), as it is evident from fig. 3. Assuming that the residual nucleus can be represented, in a simplified picture, as a "nuclear lense", from fig. 2 it can be deduced that modifying the size of the lense changes the diffractive shape of the beam of particles scattered at large angles. However, the average slope, which can be identified as the tangent to the distribution in the secondary maxima, is not modified. Moreover, a regular oscillatory pattern is due to interference among the fluxes of particles scattered by a discrete (periodical) structure of scatterers, typically pointlike sources or a lattice. Diffraction from a continuous structure would cause a distribution with a single central maximum. In the case of $\left(\mathrm{e}, \mathrm{e}^{\prime} \mathrm{p}\right)$, the obvious identification follows between the structure of scatterers and the nucleons inside the residual nucleus. But in the formalism leading to eq. (5) there is no signature of the many-body aspect of the residual interaction. The optical model is, in fact, a mean-field approximation to the problem of FSI with smoothly varying properties.

However, the diffuseness $a$ of the Woods-Saxon well (see eq. (11)) is the only parameter that introduces into the problem a dimensional length of the order of the nucleon size, which is in turn very similar to the range of nucleon-nucleon correlations. Inspection of fig. 4, where $S_{\mathrm{s} 1 / 2}^{\mathrm{D}}$ is calculated in the same conditions as in fig. 2, shows that varying $a$ not only modifies the size, but also the average slope of the angular distribution. The dashed line here corresponds to the dashed line in fig. 2. It is evident that largeangle emissions are largely affected by the nucleon-nucleon interactions taking place in the nuclear surface. Also the short-distance structure of the internal nuclear medium is important, but small volumes in the nuclear interior can be considered roughly isotropic and unable to select a preferred direction (as it is usually assumed in the Local Density Approximation).

By combining the previous observations about the sensitivity of the results to $R$ and $a$, we can deduce first that a small spatial region of size $a$ on the nuclear surface is responsible for the overall feature of the large-angle 
distribution in momentum space. Secondly, in eq. (15) the surface oscillations of $\rho(r)$ produce high-frequency components in momentum space, among which only those close to $\boldsymbol{p}_{\mathrm{m}}$ are emphasized in the Fourier transform. This corresponds to selecting only two surface regions of size $a$, which can contribute to the emission of a nucleon with initial missing momentum $\boldsymbol{p}_{\mathrm{m}}$. They can be identified through the intersection between the direction $\hat{p}_{\mathrm{m}}$ and the nuclear surface. The diffractive pattern can, therefore, be interpreted as the quantum interference between the fluxes emerging from these two regions. In fact, from fig. 2 it turns out that the finer details of the oscillations in momentum space are sensitive to the nuclear size $R$, or equivalently to the relative distance between the two regions, which is much bigger than their size $a$.

From this picture the findings in ref. [36] are confirmed that, by a suitable modification of $R, a$ in the optical potential in a way compatible with the constraints dictated by phase-shift analysis, most details of final-state rescattering in (e,e'p) at large angles can be effectively reproduced by a mean-field optical model.

Finally, it must be stressed that for all these results it is crucial that the residual nucleus be in a well defined state. Only in this case its internal structure can be coherently tested by the ejectile. Energy-integrated distributions (like semi-inclusive (e, $\mathrm{e}^{\prime} \mathrm{p}$ ) reactions [29, 37]) can test by definition only the average behaviour of the emitted proton, thus leading to very different angular shapes.

\section{Conclusions}

It has been shown elsewhere [33, 34] that for the ${ }^{12} \mathrm{C}\left(\mathrm{e}, \mathrm{e}^{\prime} \mathrm{p}\right){ }^{11} \mathrm{~B}_{\mathrm{s} 1 / 2}$ reaction at proton momenta $1 \leq p^{\prime} \leq 4 \mathrm{GeV} / \mathrm{c}$ (relevant to the planned experiments at CEBAF) the eikonal approximation to the scattering wave of the ejectile produces angular distributions very similar to the ones obtained when the complete second-order differential equation is solved up to 120 partial waves. Assuming this approximation as a reliable one, it has been here demonstrated that the large-angle part of this distribution and the corresponding one for

${ }^{11} \mathrm{~B}_{\mathrm{s} 1 / 2}(\mathrm{p}, \mathrm{p})^{11} \mathrm{~B}_{\mathrm{s} 1 / 2}$ elastic scattering have a universal feature corresponding to a coherent diffractive scattering of the outgoing proton from the ${ }^{11} \mathrm{~B}_{\mathrm{s} 1 / 2}$ excited nucleus. This is due to the dominance of FSI in this kinematical 
region. Because of the nontrivial differences between the two reactions, the comparison is meaningful only for missing momenta with a large transverse component with respect to the momentum transfer $\boldsymbol{q}$. With these constraints, it is argued that it may represent a more convenient tool to disentangle effects due to the (hard) electromagnetic vertex from (exotic) effects related to the propagation of the struck hadron through the nuclear medium. While the small-angle part of the distribution is affected mainly by the single-particle momentum distribution of the emitted proton when in its bound state (already accessible in the PWIA approximation), the large-angle part shows a marked sensitivity to the dimensional parameters of the well of the residual potential, particularly to its surface thickness which is related to the dimensional scale of the short-range nucleon-nucleon interaction.

We would like to thank O. Benhar, S. Boffi, S. Jeschonnek, N.N. Nikolaev and S. Simula for many stimulating discussions.

\section{References}

[1] A.A. V.V. in: J. Arvieux and E. De Sanctis, eds., Conference Proceedings of the Italian Physical Society: the ELFE Project, an Electron Laboratory for Europe (Editrice Compositori, Bologna, 1993).

[2] A. Saha (spokesperson), CEBAF Proposal No. E-91-006;

R. Milner (spokesperson), CEBAF Proposal No. E-91-007;

D.F. Geesaman (spokesperson), CEBAF Proposal No. E-91-013.

[3] I. Bobeldijk et al., Phys. Rev. Lett. 73 (1994) 2684.

[4] K.I. Blomqvist et al., Phys. Lett. B344 (1995) 85.

[5] C. Mahaux and R. Sartor, Adv. Nucl. Phys. 20 (1991) 1.

[6] S.C. Pieper, R.B. Wiringa and V.R. Pandharipande, Phys. Rev. C46 (1992) 1741.

[7] M. Radici, S. Boffi, S.C. Pieper and V.R. Pandharipande, Phys. Rev. C50 (1994) 3010. 
[8] H. Müther and W.H. Dickhoff, Phys. Rev. C49 (1994) R17.

[9] A. Picklesimer, J.W. Van Orden and S.J. Wallace, Phys. Rev. C32 (1985) 1312.

[10] L.L. Frankfurt, G.A. Miller and M.I. Strikman, Annu. Rev. Nucl. Part. Sci. 45 (1994) 501;

N.N. Nikolaev, Int. J. Mod. Phys. E3 (1994) 1;

A.Bianconi, S.Boffi and D.E.Kharzeev, Yadernaya Fizika 57 (1994) 1732.

[11] R.J. Glauber in: W. Brittain and L.G. Dunham, eds., Lectures in Theoretical Physics 1 (Interscience Publ., New York, 1959);

R.J. Glauber and G. Matthiae, Nucl. Phys. B21 (1970) 135.

[12] R.H. Bassel and C. Wilkin, Phys. Rev. 174 (1968) 1179.

[13] G.D. Alkhazov, S.I. Belostotsky and A.A. Vorobyev, Phys. Rep. 42 (1978) 89.

[14] S.J. Wallace, Phys. Rev. C12 (1975) 179.

[15] S. Frullani and J. Mougey, Adv. Nucl. Phys. 13 (1984) 1.

[16] S. Boffi, C. Giusti and F.D. Pacati, Phys. Rep. 226 (1993) 1.

[17] S. Boffi, F. Cannata, F. Capuzzi, C. Giusti and F.D. Pacati, Nucl. Phys. A379 (1982) 509.

[18] S. Gardner and J. Piekarewicz, Phys. Rev. C50 (1994) 2822.

[19] S. Boffi, C. Giusti, F.D. Pacati and S. Frullani Nucl. Phys. A319 (1979) 461.

[20] F.G. Perey and B. Buck, Nucl. Phys. 32 (1962) 353;

F. Capuzzi, Lecture Notes in Physics 55 (1976) 20;

H. Fiedeldey, Nucl. Phys. 77 (1966) 149.

[21] J.R. Comfort and B.C. Karp, Phys. Rev. C21 (1980) 2162. 
[22] R.D. Amado, J. Piekarewicz, D.A. Sparrow and J.A. McNeil, Phys. Rev. C28 (1983) 1663.

[23] E. Rost, J.R. Shepard and D. Murdock, Phys. Rev. Lett. 49 (1982) 448.

[24] V.N. Gribov, Sov. Phys. JEPT 29 (1969) 483.

[25] G.R. Farrar, H. Liu, L.L. Frankfurt and M.I. Strikman, Phys. Rev. Lett. 61 (1988) 686;

B.K. Jennings and G.A. Miller, Phys. Rev. D44 (1991) 692;

B.Z. Kopeliovich and B.G. Zakharov, Phys. Rev. D44 (1991) 3466;

W.R. Greenberg and G.A. Miller, Phys. Rev. D47 (1993) 1865.

[26] C. Ciofi degli Atti and S. Simula, Phys. Lett. B325 (1994) 276.

[27] N.C.R. Makins et al. (the NE18 Collaboration), Phys. Rev. Lett. 72 (1994) 1986.

[28] V.R. Pandharipande and S.C. Pieper, Phys. Rev. C45 (1992) 791.

[29] N.N. Nikolaev, A. Szczurek, J. Speth, J. Wambach, B.G. Zakharov and V.R. Zoller, Phys. Rev. C50 (1994) R1296;

N.N. Nikolaev, A. Szczurek, J. Speth, J. Wambach, B.G. Zakharov and V.R. Zoller, Nucl. Phys. A582 (1995) 665.

[30] A. Kohama, K. Yazaki and R. Seki, Nucl. Phys. A536 (1992) 716.

[31] L.L. Frankfurt, E.J. Moniz, M.M. Sargsyan and M.I. Strikman, Phys. Rev. C51 (1995) 3435.

[32] C. Lechanoine-LeLuc and F. Lehar, Rev. Mod. Phys. 65 (1993) 47.

[33] A. Bianconi and M. Radici, Phys. Rev. C53 (1996) R563.

[34] A. Bianconi and M. Radici, Phys. Lett. B363 (1995) 24.

[35] B. Kopeliovich, in Proceedings from Workshop on Options for Color Coherence/Transparency Studies at CEBAF (May 22-23 1995, Newport News), p. 180. 
[36] D.G. Ireland, L. Lapikás and G. van der Steenhoven, Phys. Rev. C50 (1994) 1626.

[37] A. Bianconi, S. Jeschonnek, N.N. Nikolaev and B.G. Zakharov, Phys. Lett. B338 (1994) 123. 


\section{Captions}

Fig. 1 - The solid line represents the distorted spectral density $S_{\mathrm{s} 1 / 2}^{\mathrm{D}}$ in the eikonal approximation for the ${ }^{12} \mathrm{C}\left(\mathrm{e}, \mathrm{e}^{\prime} \mathrm{p}\right){ }^{11} \mathrm{~B}_{\mathrm{s} 1 / 2}$ reaction at $p^{\prime}=q=4$ $\mathrm{GeV} / \mathrm{c}$ for various values of transverse missing momentum and for a purely imaginary optical potential with depth $W=50 p^{\prime} / p_{\mathrm{o}} \mathrm{MeV}$, with $p_{\mathrm{o}}=1.4 \mathrm{GeV} / \mathrm{c}$. The bound state is derived from the potential of Comfort and Karp [21]. The short-dashed line shows the result when the further approximation of eq. (18) is applied (see text). The longdashed line refers to the transition probability $S^{\mathrm{DD}}$ of eq. (15) (see text) for the ${ }^{11} \mathrm{~B}_{\mathrm{s} 1 / 2}(\mathrm{p}, \mathrm{p}){ }^{11} \mathrm{~B}_{\mathrm{s} 1 / 2}$ reaction in the same kinematics.

Fig. 2 - The distorted spectral density $S_{\mathrm{s} 1 / 2}^{\mathrm{D}}$ for the ${ }^{12} \mathrm{C}\left(\mathrm{e}, \mathrm{e}^{\prime} \mathrm{p}\right){ }^{11} \mathrm{~B}_{\mathrm{s} 1 / 2}$ reaction in the same kinematical conditions as in fig. 1. The dashed line corresponds to the solid line in fig. 1. The upper $\left(\right.$ at $\left.\gamma \sim 6^{\circ}\right)$ solid lines are obtained when reducing the well radius of the optical potential by $17 \%$ and $8 \%$; the lower one when increasing it by $8 \%$.

Fig. 3 - The distorted spectral density $S_{\mathrm{s} 1 / 2}^{\mathrm{D}}$ for the ${ }^{12} \mathrm{C}\left(\mathrm{e}, \mathrm{e}^{\prime} \mathrm{p}\right)^{11} \mathrm{~B}_{\mathrm{s} 1 / 2}$ reaction in the same kinematical conditions as in fig. 1, but with a variable imaginary depth $W$ of the optical potential. The dashed line is obtained with $W=150 \mathrm{MeV}$, which corresponds approximately to the solid line in fig. 1. The upper and lower (at $\gamma=0^{\circ}$ ) solid lines correspond to $W=100$ and $200 \mathrm{MeV}$, respectively. 
Fig. 4 - The distorted spectral density $S_{\mathrm{s} 1 / 2}^{\mathrm{D}}$ for the ${ }^{12} \mathrm{C}\left(\mathrm{e}, \mathrm{e}^{\prime} \mathrm{p}\right){ }^{11} \mathrm{~B}_{\mathrm{s} 1 / 2}$ reaction in the same kinematical conditions as in fig. 1. The dashed line corresponds to the solid line in fig. 1. The lower (at $\gamma \sim 10^{\circ}$ ) solid line is obtained when reducing the diffuseness of the optical potential by $50 \%$; the upper one when increasing it by $50 \%$. 


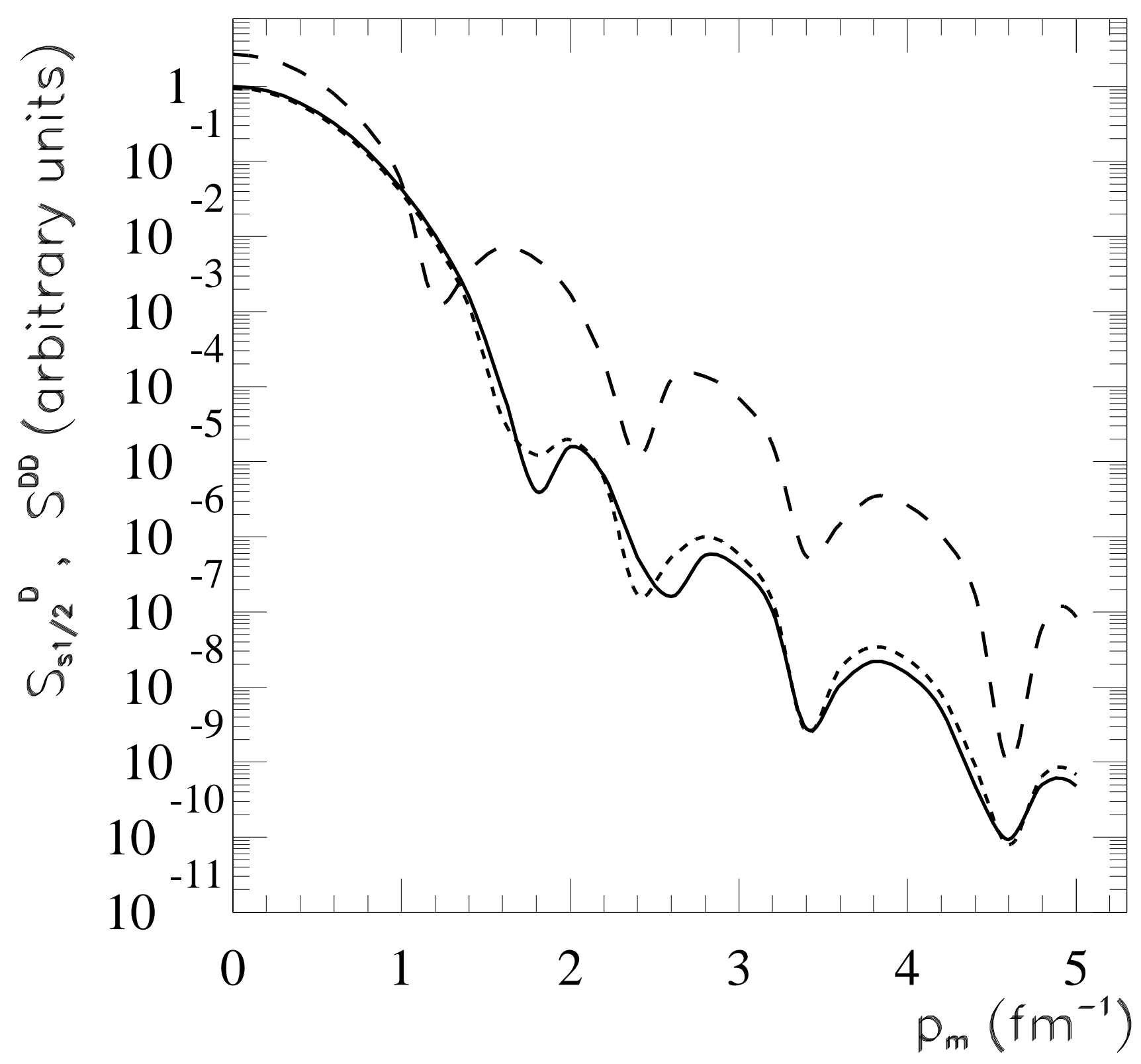

Fig. 1 


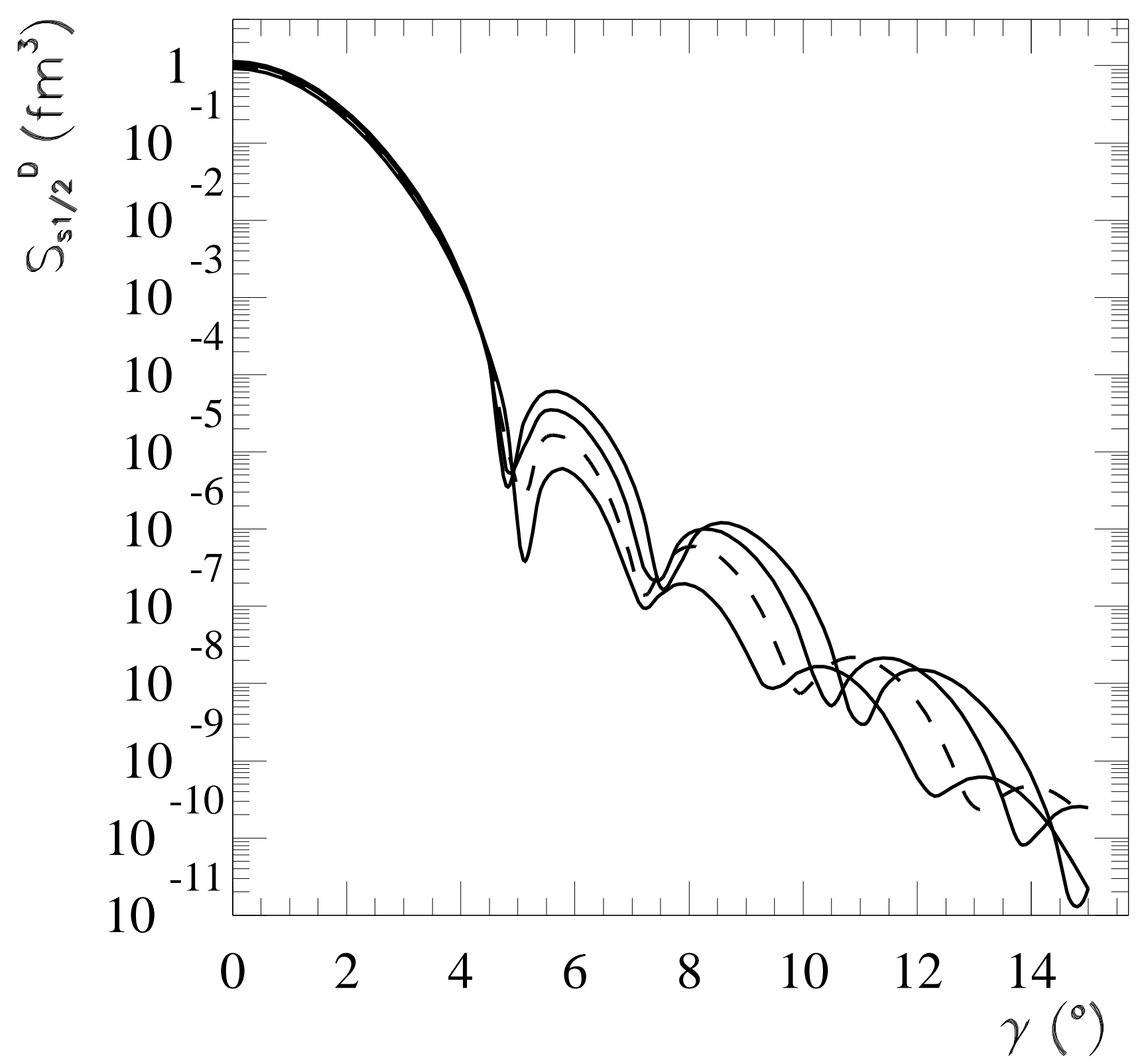

Fig. 2 


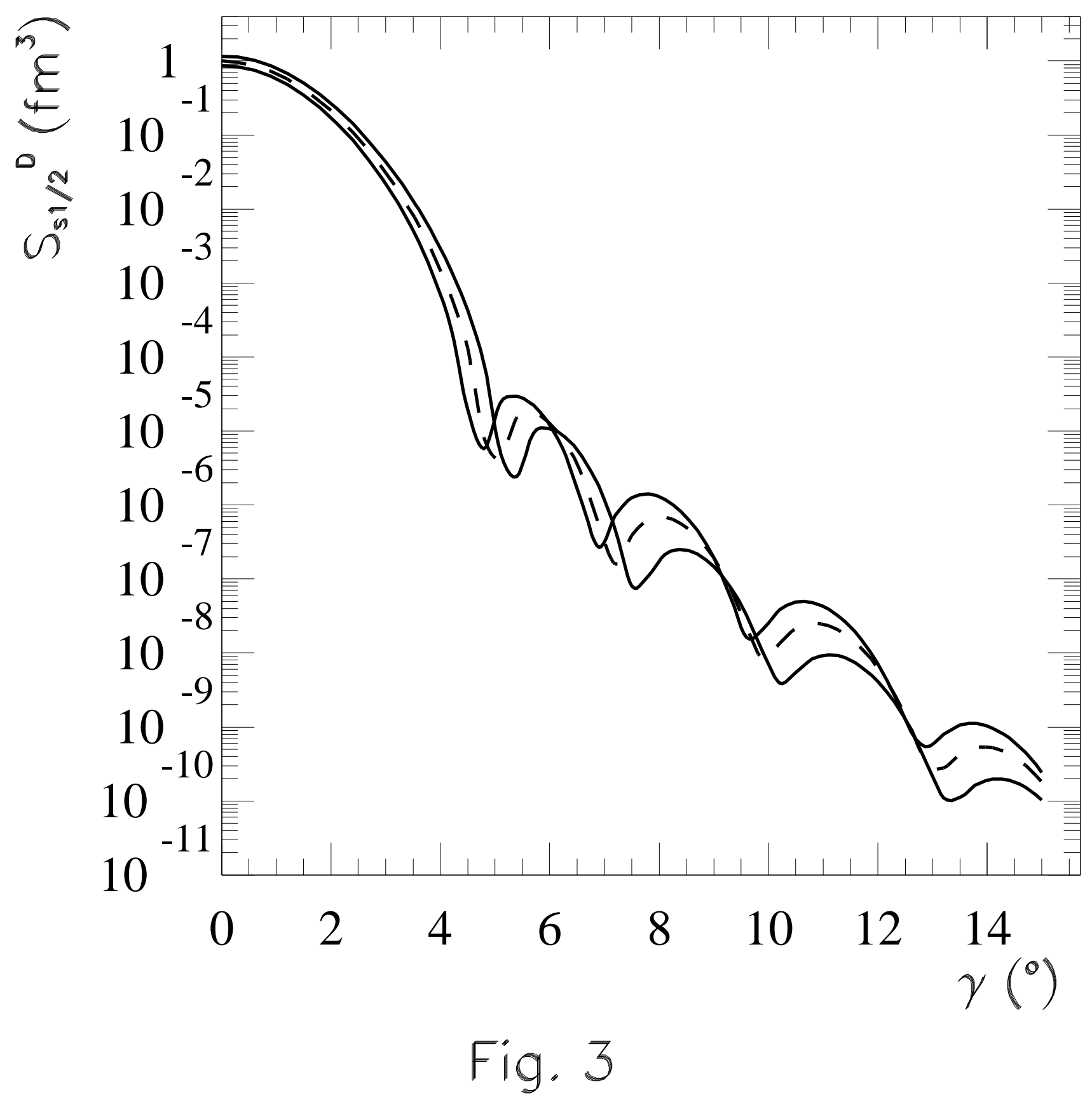




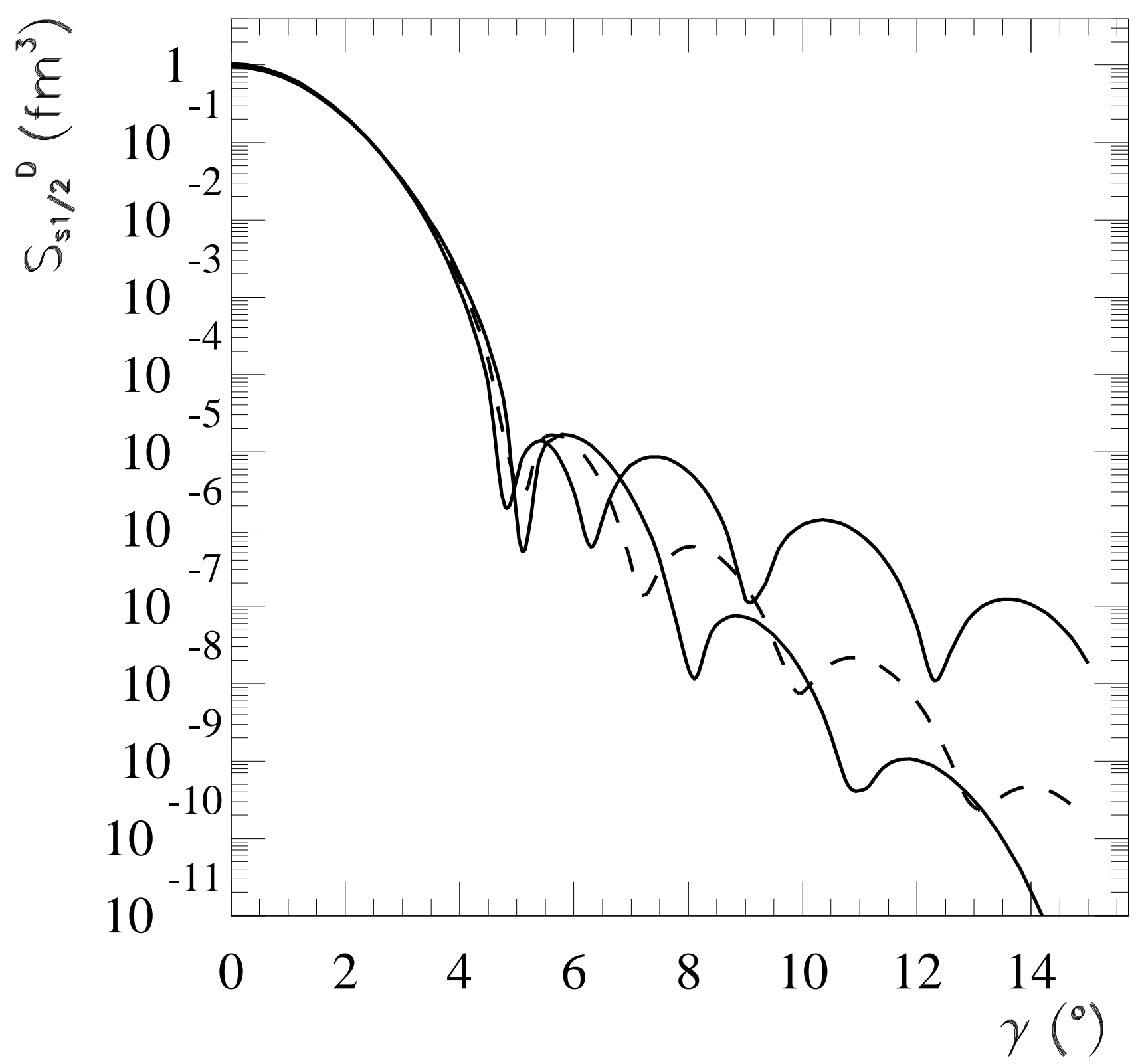

Fig. 4 